\title{
Restored expression of the tumor suppressor gene RUNX3 reduces cancer stem cells in hepatocellular carcinoma by suppressing Jagged1-Notch signaling
}

\author{
SHIN-ICHI NISHINA ${ }^{1}$, HIDENORI SHIRAHA ${ }^{1}$, YUTAKA NAKANISHI ${ }^{1}$, SHIGETOMI TANAKA $^{1}$, \\ MINORU MATSUBARA $^{1}$, NOBUYUKI TAKAOKA ${ }^{1}$, MASAYUKI UEMURA ${ }^{1}$, SHIGERU HORIGUCHI ${ }^{1}$, \\ JUNRO KATAOKA $^{1}$, MASAYA IWAMURO ${ }^{1}$, TAKAHITO YAGI ${ }^{2}$ and KAZUHIDE YAMAMOTO ${ }^{1}$
}

Departments of ${ }^{1}$ Gastroenterology and Hepatology, and ${ }^{2}$ Gastroenterological Surgery, Transplant, and Surgical Oncology, Okayama University Graduate School of Medicine and Dentistry, 2-5-1 Shikata-cho, Kita-ku, Okayama 700-8558, Japan

Received December 1, 2010; Accepted February 28, 2011

DOI: $10.3892 / o r .2011 .1336$

\begin{abstract}
Runt-related transcription factor 3 (RUNX3) is a candidate tumor suppressor gene that is downregulated in various cancers. In the present study, we analyzed the regulatory function of RUNX3 on Jagged-1 (JAG1) expression and cancer stem cell (CSC) signaling in hepatocellular carcinoma (HCC). Eleven HCC cell lines and 30 human HCC tissues were used. RUNX3 and JAG1 expression levels were analyzed by immunoblotting and immunohistochemistry. Ectopic RUNX3 expression was induced by introducing RUNX3 cDNA into the RUNX3-negative HCC cell line Hep3B and Huh7 cells. Furthermore endogenous RUNX3 expression was knocked down by RUNX3 siRNA in SK-Hep-1 cells. In order to analyze JAG1 transcriptional regulation, we conducted reporter assays, chromatin immunoprecipitation (ChIP) assays and electrophoretic mobility shift assays (EMSAs). Tumorigenicity was analyzed using a SCID mouse liver injection model. An inverse correlation was observed between RUNX3 expression and JAG1 expression in most HCC cell lines and tissues. Restoring RUNX3 expression decreased the expression of JAG1 in Hep3B and Huh7 cells, whereas JAG1 expression was upregulated in RUNX3 siRNA-treated SK-Hep-1 cells. Reporter assays, ChIP assays and EMSAs revealed that RUNX3 directly bound to the transcriptional regulatory region of JAG1 and suppressed JAG1 transcription. Moreover, RUNX3 restoration downregulated CSCs by suppressing JAG1-mediated Notch signaling. The tumorigenic capacity of RUNX3-expressing Hep3B cells was lower compared to that of control Hep3B cells. RUNX3 expression suppressed JAG1 expression and
\end{abstract}

Correspondence to: Dr Shin-Ichi Nishina, Department of Gastroenterology and Hepatology, Okayama University, 2-5-1 Shikata-cho, Kita-ku, Okayama 700-8558, Japan

E-mail: shin12470123@yahoo.co.jp

Key words: runt-related transcription factor 3, Jagged-1, Notch signaling, cancer stem cell, hepatocellular carcinoma resulted in downregulation of tumorigenesis by suppression of JAG1-mediated CSCs.

\section{Introduction}

Hepatocellular carcinoma (HCC) is the third most frequent cause of cancer-related death worldwide, and its incidence rate is increasing (1-3). Patients diagnosed with HCC have a poor prognosis because of the aggressive nature of the disease $(1,4)$. Although various therapeutic strategies have been developed, such as operation, ablation and chemotherapy, therapies to treat patients with advanced HCC are lacking. There is an urgent need to elucidate the pathologic mechanism underlying molecular-targeted therapies in HCC. Many studies have considered that the dysfunction of oncogenes and tumor suppressor genes, reactivation of developmental pathways, and activation of growth factors and their receptors play major roles in the development and progression of $\mathrm{HCC}(1,5)$. Further, the expression of a number of tumor suppressor genes is attenuated by a combination of genetic and epigenetic events, including loss of an allele, mutation, or promoter methylation $(6,7)$. The loss of heterozygosity ( $\mathrm{LOH}$ ) on chromosome 1p36 was reported as one of the most frequent $\mathrm{LOH}$ in $\mathrm{HCC}(7,8)$. $\mathrm{LOH}$ on chromosome $1 \mathrm{p} 36$ suggests the presence of important tumor suppressor genes on that chromosome. It was reported that Runt-related transcription factor 3 (RUNX3), located on chromosome 1p36, correlates with tumorigenesis and progression of gastric cancer (9). RUNX3 is an apoptotic factor located downstream from TGF- $\beta$ and the cell differentiation mediator of conditions such as intestinal metaplasia of the gastric mucosa $(10,11)$. Decreased RUNX3 expression accounts for $\sim 45-60 \%$ of human gastric cancers and has been shown in various human malignancies such as those of the colon, lung, pancreas and bile duct (12-16). It has been reported that RUNX3 gene expression is decreased in $30-80 \%$ of HCC tissues due to LOH and methylation of its promoter lesion $(6,17)$. RUNX3 is reported to be a multifunction transcription factor; therefore, the functions of RUNX3 in HCC need to be elucidated. In the 
present study, we demonstrated that the restoration of RUNX3 expression repressed Jagged-1 (JAG1)-Notch signaling.

The Notch signaling pathway is an evolutionarily conserved intercellular signaling mechanism that affects not only many differentiation processes and cell-fate determination during embryonic and postnatal development, but also the regulation of self-renewing tissues and stem cell maintenance (18). Four Notch receptors (Notch-1 to Notch-4) and 5 ligands (JAG1 and -2 , and $\delta$-like-1 -3 and -4) have been described in mammals (19). The activation of Notch signaling is mediated by the interaction of bordering cells via cell-to-cell contact of the membrane-associated Notch receptor and ligand. Although Notch signaling has been associated mostly with oncogenic and growth-promoting roles, depending on the tissue type, it can also function as a tumor suppressor $(20,21)$. It remains controversial whether Notch functions in HCC as an oncogene or tumor suppressor. On the other hand, JAG1-dependent Notch signaling is dispensable for hematopoietic stem cell self-renewal and differentiation (22). Others have reported that Notch signaling regulates cancer stem cells (CSCs) in breast cancer, colon cancer and melanoma (23-25).

The CSC hypothesis has received enormous attention from researchers. Initial studies on leukemia provided a paradigm for the general CSC model, suggesting that a similar model existed for solid tumors with CSCs at the top of a hierarchy $(26,27)$. The dysregulated self-renewal of hepatic stem cells may cause hepatocarcinogenesis.

In the current study, we show that loss of RUNX3 causes aberrant JAG1 expression and induces CSCs in HCC. Our investigation provides a novel molecular mechanism in HCC and suggests that the aberrant expression of JAG1 influences stem cell dysfunction and hepatocarcinogenesis.

\section{Materials and methods}

HCC tissues and immunohistochemistry. Thirty patients, consisting of 23 men aged 18-76 years (average, 58.3 years) and 7 women aged 54-82 years (average, 64.7 years) at the time of hepatic resection, were included in this study. HCC tissues along with adjacent liver tissues were used for analysis. Human HCC tissues were obtained through a written informed consent process that adhered to the stringent ethical criteria of the Okayama University Graduate School of Medicine, Dentistry and Pharmaceutical Sciences. Immunohistochemistry was performed on formalin-fixed, paraffin-embedded tissue sections that were dewaxed and dehydrated. After rehydration, endogenous peroxidase activity was blocked for $10 \mathrm{~min}$ in a methanol solution containing $0.3 \%$ hydrogen peroxide. After antigen retrieval in a citrate buffer, the sections were blocked for $30 \mathrm{~min}$ at $4^{\circ} \mathrm{C}$. The sections were probed with 1:100 mouse monoclonal anti-RUNX3 antibody (ab49117; Abcam, Cambridge, MA) and 1:250 polyclonal anti-JAG1 antibody (sc-34473; Santa Cruz Biotechnology, Santa Cruz, CA). The primary antibody was detected using a biotinylated antibody (Dako Japan, Tokyo, Japan). The signal was amplified by avidin-biotin complex formation and developed with diamino-benzidine followed by counterstaining with hematoxylin, dehydration in alcohol and xylene, and mounting. The sections were scored for RUNX3 and JAG1 expression with a 4-titer scale as follows: 0 , negative; 1 , weak signal; 2 , interme- diate signal; and 3, strong signal (28). All sections were scored independently by 2 blinded observers. All discrepancies in scoring were reviewed, and a consensus was reached.

Cell lines and cell culture. Eleven human HCC cell lines were utilized. HCC cell lines HepG2, Hep3B, PLC/PRF/5 (PLC) and Sk-Hep-1 were obtained from the American Type Culture Collection (Manassas, VA). HCC cell lines Huh1, Huh7, JHH1, JHH2, JHH4, HLE and HLF were obtained from the Health Science Research Resources Bank (Osaka, Japan). HepG2, Hep3B, Huh1, Huh7, JHH4, HLE, HLF, PLC and Sk-Hep-1 cells were maintained in Dulbecco's modified Eagle's medium (DMEM) (Invitrogen, Carlsbad, CA). JHH1 and JHH2 were cultured in William's medium E (Invitrogen). DMEM and William's medium E were supplemented with $10 \%$ heatinactivated fetal bovine serum (FBS) (Sigma, St. Louis, MO), $1 \%$ non-essential amino acids (Sigma), 1\% sodium pyruvate (Sigma), and 1\% penicillin/streptomycin solution (Sigma). Cells were cultured at $37^{\circ} \mathrm{C}$ in a humidified atmosphere of $5 \% \mathrm{CO}_{2}$ and $95 \%$ air. In some experiments, $25 \mu \mathrm{M} \gamma$-secretase inhibitor (N-[N-(3,5-difluorophenacetyl-1-alanyl)]-S-phenylglycine t-butyl ester [DAPT]) (Calbiochem, San Diego, CA) was added every 3 to 4 days to the culture medium, and the controls consisted of cultures treated with the same concentration of vehicle (DMSO).

Immunoblot analysis. Cells were plated in 6-well tissue culture plastic dishes and grown to confluence. The cells were washed twice with cold phosphate-buffered saline (PBS) and lysed in $150 \mu \mathrm{l}$ of sample buffer [100 mM Tris- $\mathrm{HCl}$ $\mathrm{pH} 6.8,10 \%$ glycerol, $4 \%$ sodium dodecyl sulfate (SDS), $1 \%$ bromophenol blue, $10 \% \beta$-mercaptoethanol]. The samples were resolved by SDS-polyacrylamide gel electrophoresis (PAGE) and transferred to an Immobilon- $\mathrm{P}^{\mathrm{TM}}$ polyvinylidene difluoride membrane (Millipore, Bedford, MA). The membranes were blocked using PVDF Blocking Reagent for Can Get Signal $^{\mathrm{TM}}$ Solution (Toyobo Life Science, Osaka, Japan) for $1 \mathrm{~h}$. The membranes were incubated with anti-RUNX3 antibody (ab40278, Abcam), anti-JAG1 antibody (no. 2620, Cell Signaling Technology, Beverly, MA), anti-Notch1 antibody (no. 2495, Cell Signaling), anti-cleaved Notch1 antibody (no. 2421, Cell Signaling), anti-HES1 antibody (AB5702, Millipore), anti-HEY1 antibody (ab22614, Abcam) and antiactin antibody (A2066, Sigma) dissolved in Can Get Signal solution A overnight at $4{ }^{\circ} \mathrm{C}$. Target proteins were probed with peroxidase-conjugated secondary antibodies dissolved in Can Get Signal solution B for $1 \mathrm{~h}$ at room temperature. The blotted proteins were detected by using an ECL plus ${ }^{\mathrm{TM}}$ kit (GE Healthcare Biosciences, Piscataway, NJ).

RUNX3 transfection. The human RUNX3 or chloramphenicol acetyltransferase (CAT) (control) constructs were cloned into the pCEP4 (Invitrogen) expression vector. The RUNX3 or CAT constructs were transfected into Hep3B cells by using FuGENE6 $^{\mathrm{TM}}$ transfection reagent (Roche Diagnostics, Basel, Switzerland). These transfected cells were designated as Hep3BRUNX3 and Hep3B-CAT cells, respectively. Forty-eight hours after transfection, cells were selected in the complete medium containing $250 \mu \mathrm{g} / \mathrm{ml}$ hygromycin (Roche). Polyclonal lines consisting of $>20$ colonies were established. At least 2 indepen- 
dent transfections and stably transfected lines were established for each construct.

Gene silencing of RUNX3 with small interfering RNA. The RUNX3 gene was knocked down by using the siRNA expression vector pSilencer2.1-U6 hygro (Applied Biosystems, Foster City, CA). Sense (5'-GATCCGTCGGAACTGAACCC ATTCTTTCAAGAGAAGAATGGGTTCAGTTCCGAGGT TTTTTGGAAA-3') and antisense (5'-AGCTTTTCCAAAAA ACCTCGGAACTGAACCCATTCTTCTCTTGAAAGAAT GGGTTCAGTTCCGACG-3') siRNA template oligonucleotides were annealed with annealing solution at $90^{\circ} \mathrm{C}$ for $3 \mathrm{~min}$ according to the manufacturer's instructions. The annealed oligonucleotides were inserted into the psilencer2.1-U6 hygro vector. RUNX3 siRNA and control siRNA expression vectors were transfected into Sk-Hep-1 cells by using FuGENE6 transfection reagent in 6-well tissue culture plastic dishes. At $48 \mathrm{~h}$ after transfection, cells were used for the following experiments.

Luciferase reporter assay. A 574-bp transcriptional regulatory region of JAG1 [transcriptional regulatory element database (TRED) promoter ID no. 113024 from -574 to -1] was cloned by polymerase chain reaction (PCR) from genomic DNA of normal human primary hepatocytes (hNHeps; Sanko Junyaku, Tokyo, Japan) (29). The 574-bp region contains the RUNX3 binding site (5'-ACCACA-3') at -145 to -140 . A transcriptional regulatory region of a different length (from -100 to -1) without the RUNX3 binding site was also cloned. These transcriptional regulatory regions of JAG1 were subcloned into the firefly luciferase reporter vector pGL4.23 (Promega, Madison, WI). A luciferase reporter vector including the mutant transcriptional regulatory region of JAG1 was also generated. The RUNX3 binding site in the transcriptional regulatory region of JAG1 was mutated to 5'-GTGTAC-3' by using the Site-Directed Mutagenesis kit (Stratagene, La Jolla, CA) according to the manufacturer's instructions. These constructs were designated as pGL4-574, pGL4-100 and pGL4-574m, respectively. All constructs were verified by sequencing.

The reporter plasmids, $8 \mu \mathrm{g}$ of the indicated pGL4.23 vector containing the transcriptional regulatory region of JAG1, $1 \mu \mathrm{g}$ of the pGL4.74 vector (Promega), which contains the Renilla luciferase gene as a transfection efficiency control, and $8 \mu \mathrm{g}$ of RUNX3/control construct per $1 \times 10^{5}$ cells were cotransfected using FuGENE6 transfection reagent. After $48 \mathrm{~h}$, cells were washed with PBS, and lysates prepared by adding $500 \mu 1$ of passive lysis buffer (Dual Luciferase ${ }^{\mathrm{TM}}$ Reporter Assay System, Promega). The activities of firefly and Renilla luciferase were measured sequentially with a luminometer (Berthold Technologies, Bad Wildbad, Germany). These experiments were performed independently 3 times with similar results. Statistical analysis was performed on luciferase activity by using JMP software (SAS Institute, Cary, NC). The significance of the data was determined using the unequal variance t-test (Student's t-test).

Electrophoresis mobility shift assay. An electrophoresis mobility shift assay (EMSA) was performed using nuclear extract prepared from Hep3B-CAT and Hep3B-RUNX3 cells plated into $10-\mathrm{cm}$ plates at $80 \%$ confluence. Nuclear extract was prepared using a nuclear extraction kit (Panomics, Redwood, CA) and stored at $-80^{\circ} \mathrm{C}$ until use. Complementary oligonucleotides corresponding to the RUNX3-binding site (5'-GGCTTTTCAACCACAAAACTCATC-3') of the transcriptional regulatory region of JAG1 were labeled with digoxigenin at the $3^{\prime}$ end and used as probes. EMSA was performed using a DIG Gel Shift kit 2nd generation (Roche) according to the manufacturer's instructions. For a supershift assay, nuclear extracts were incubated with an anti-RUNX3 antibody (Abcam) for $30 \mathrm{~min}$ before electrophoresis. Binding reactions were resolved by running extracts on a $10 \%$ polyacrylamide gel for $80 \mathrm{~min}$ at $100 \mathrm{~V}$, followed by transfer onto a nylon membrane for $40 \mathrm{~min}$ at $0.4 \mathrm{~A}$. After blotting, nylon membranes were cross-linked with ultraviolet light using a Stratalinker ${ }^{\mathrm{TM}}$ UV crosslinker (Stratagene). Digoxigenin signals were visualized by chemiluminescent detection by using the DIG DNA detection kit (Roche).

Chromatin immunoprecipitation assay. Chromatin immunoprecipitation (ChIP) assays were performed using the ChIP assay kit (17-295, Upstate Biotechnology, Lake Placid, NY) according to the manufacturer's protocol. Briefly, $2 \times 10^{6}$ cells were cross-linked by formaldehyde, lysed and fragmented by sonication. Prior to crosslinking, an aliquot of the cells was removed to analyze input chromatin DNA. Cell extracts were pretreated with agarose beads before incubation with an anti-RUNX3 monoclonal antibody (ab40278, Abcam) or a non-specific antibody control overnight. After incubation with antibodies, the complex was collected with agarose beads, and crosslinking between protein and DNA was reversed. Immunoprecipitated DNA was analyzed by PCR by using JAG1 ChIP primers 5'-AGTCTGTTCTGGTAATCGGGGTAT-3' and 5'-CAGTGGTGTTTATTCAAGCAGTAT-3'.

Flow cytometry analysis. For flow cytometry, cells were detached with Accutase ${ }^{\mathrm{TM}}$ (Chemicon International, San Diego, $\mathrm{CA}$ ) and washed twice in the staining solution containing $\mathrm{Ca}^{2+}$ and $\mathrm{Mg}^{2+}$-free PBS with $1 \mathrm{mM}$ EDTA, 25 mM HEPES (pH 7.0; Sigma), and $1 \%$ FBS. Cells were stained live in staining solution containing anti-human CD44-fluorescein isothiocyanate (FITC; Beckman Coulter, Miami, FL), CD90-FITC (Beckman Coulter), CD133-allophycocyanin (APC; Miltenyi Biotech, Bergisch Gladbach, Germany), and epithelial cell adhesion molecule (EpCAM)-APC (Miltenyi Biotech) for $30 \mathrm{~min}$ at $4^{\circ} \mathrm{C}$. Isotype-matched mouse immunoglobulins served as controls. Labeled cells were analyzed on a FACSAria (BectonDickinson, San Jose, CA). Dead cells were eliminated using $0.25 \mu \mathrm{g} / \mathrm{ml}$ propidium iodide (PI) (Dojindo Laboratories, Kumamoto, Japan). A minimum of 500,000 viable cell events were recorded per sample. The data were analyzed using FlowJo software (Treestar, San Carlos, CA).

HCC xenografts in severe combined immunodeficiency mice. The care and treatment of mice were in accordance with the guidelines of the IACUC at the Okayama University Faculty of Medicine. Severe combined immunodeficiency (SCID) mice (8 weeks of age) were purchased from Japan SLC (Hamamatsu, Japan). Then, $2 \times 10^{6}$ of either Hep3B-CAT or Hep3B-RUNX3 cells were resuspended in $100 \mu \mathrm{l}$ of culture medium and injected into the livers of SCID mice. Eight weeks after the injection, 


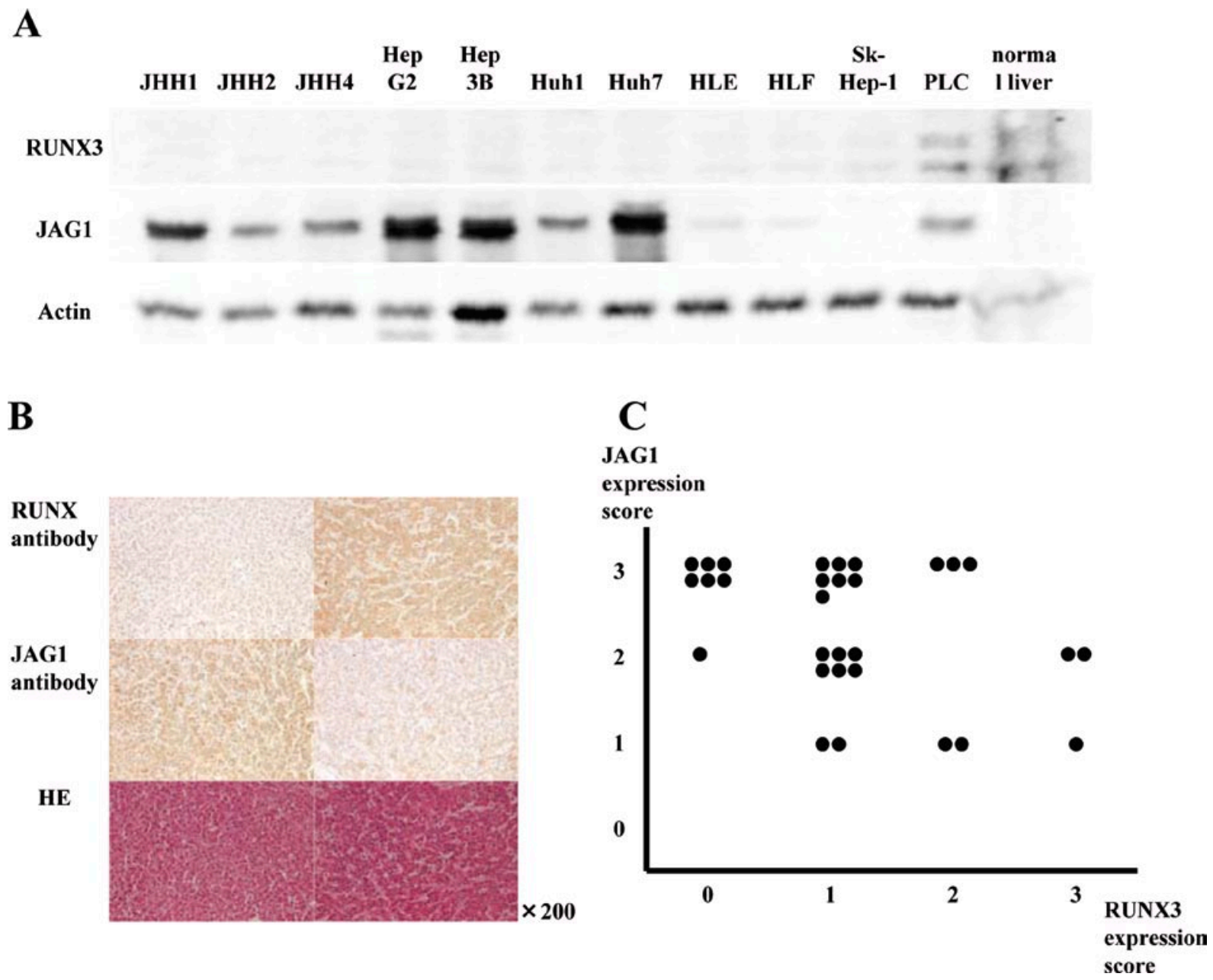

Figure 1. RUNX3 and JAG1 expression in HCC. (A) RUNX3, JAG1 and actin protein expression levels were analyzed by immunoblotting in HCC cell lines and normal liver tissue. Shown here are representative gels from 3 independent studies. (B) Representative images of RUNX3 and JAG1 staining in HCC tissues by immunohistochemistry. Representative images are from immunohistochemical analysis for RUNX3 (upper) and JAG1 (middle) expression and histology with hematoxylin and eosin staining (lower) in HCC tissues. Three sets of consecutive tissue sections represent negative (left upper) and strong (right upper) RUNX3 expression. (C) RUNX3 and JAG1 expression levels were analyzed by immunohistochemistry in human HCC tissues. The sections were scored for RUNX3 and JAG1 expression with a 4-titer scale as follows: 0, negative; 1, weak signal; 2, intermediate signal; and 3, strong signal. The inverse correlation between RUNX3 expression and JAG1 expression was assessed by Spearman correlation coefficient analysis. $\mathrm{p}<0.05 ; \mathrm{r}=-0.409$.

the SCID mice were sacrificed, and the tumors were weighed. Comparison of tumor weight was performed by two-sample (control Hep3B-CAT vs. Hep3B-RUNX3) unequal variance $t$-test (Student's $t$-test) for the significance level $p<0.001$ by using JMP software.

\section{Results}

Inverse correlation between RUNX3 expression and JAG1 expression in HCC cell lines and tissues. Immunoblot analysis was performed to evaluate RUNX3 and JAG1 expression in 11 HCC cell lines and normal human liver tissue. An inverse correlation between RUNX3 expression and JAG1 expression was observed in most of the HCC cell lines (Fig. 1A). Only in PLC cells was simultaneous expression of RUNX3 and JAG1 observed. To determine if there was a relationship between RUNX3 and JAG1 expression levels in HCC tissues, these levels were compared with those in the corresponding tumorfree resection margins (Fig. 1B). A strong or intermediate signal for JAG1 expression was found in 25/30 (83.3\%) of HCC tissues. The scores for JAG1 immunoreactivity were plotted against those for RUNX3 in HCC tissues (Fig. 1C). In general, high JAG1 expression scores were observed in HCC tissues with low RUNX3 expression. An inverse correlation between RUNX3 expression and JAG1 expression was observed in HCC tissues. (Spearman correlation coefficient $=-0.409$; $\mathrm{p}<0.05)$.

Ectopic RUNX3 expression repressed JAG1 expression and its signaling in HCC cell lines. Ectopic RUNX3 expression was evaluated in endogenous RUNX3-negative Hep3B and Huh7 cells. As shown in Fig. 2A, these cells expressed RUNX3 protein. Restoring RUNX3 expression decreased the expression of JAG1 in both Hep3B and Huh7 cells. Notch signaling activation was observed in normal and control CAT-transfected Hep3B and Huh7 cells, as demonstrated by cleaved Notch1 expression and induction of its target genes Hes1 and Heyl (Fig. 2A). In ectopic RUNX3-expressing Hep3B and Huh7 cells, JAG1, cleaved Notch1, Hes1 and Heyl expression levels were markedly decreased, indicating that ectopic RUNX3 
A

Hep3B Huh7

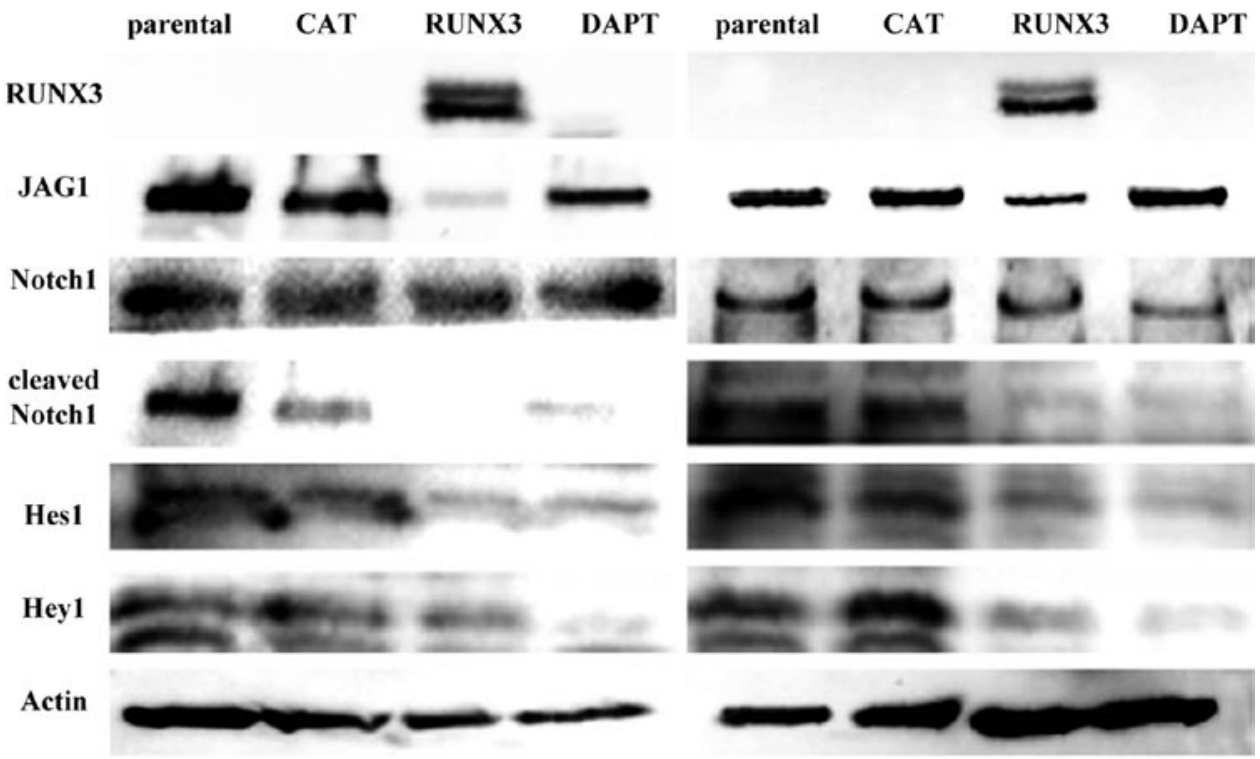

B

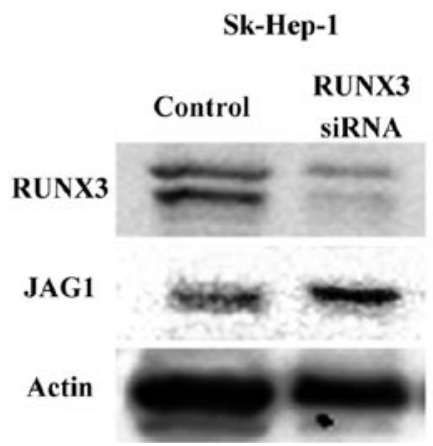

Figure 2. The effect of ectopic RUNX3 expression on JAG1 expression and Notch signaling molecules. (A) CAT (control) and RUNX3 constructs were introduced into Hep3B and Huh7 cells. Polyclonal stably expressing cell lines were selected in the presence of hygromycin. DAPT was added every 3-4 days to culture medium $(25 \mu \mathrm{M})$. Immunoblot analysis was performed with anti-RUNX3, anti-JAG1, anti-Notch1, anti-cleaved Notch1, anti-Hes1, anti-Hey1 and anti-actin antibodies. Shown here are representative blots from 3 or more independent experiments. (B) Endogenous RUNX3 expression was knocked down by using RUNX3 siRNA in Sk-Hep-1 cells. After treatment with JAG1 siRNA duplexes, immunoblot analysis was performed with anti-RUNX3, anti-JAG1 and anti-actin antibodies. Shown here are representative blots from at least 3 independent experiments.

expression negatively regulated the JAG1-Notch signaling pathway. To confirm that RUNX3 negatively regulated JAG1 expression, endogenous RUNX3 expression was knocked down by using RUNX3 siRNA in the RUNX3-expressing HCC cell line Sk-Hep-1. Endogenous JAG1 expression in Sk-Hep-1 cells was very weak, while the expression of JAG1 was upregulated in RUNX3 siRNA-treated Sk-Hep-1 (Fig. 2B).

Repression of JAG1 transcriptional activity by RUNX3. To determine whether RUNX3 regulates the expression of JAG1 at the transcriptional level, we conducted a JAG1 reporter assay (Fig. 3A). We retrieved the transcriptional regulatory region of JAG1 containing the RUNX3 binding site by using TRED. A RUNX3 binding site (5'-ACCACA-3') was found in the transcriptional regulatory region of JAG1 (TRED promoter ID113024 from -145 to -140$)$. JAG1 reporter vectors pGL4-574, pGL4-100 and pGL4-574m were generated and transfected in Hep3B cells. The JAG1 transcriptional activity in RUNX3-expressing Hep3B cells was weaker than that in control CAT-transfected Hep3B cells. Mutations in the RUNX3 binding site in reporter constructs containing the $-574 /-1$ or shorter $-100 /-1$ fragment without the RUNX3 binding site had no effect on RUNX3-dependent repression (Fig. 3A). Thus, the $-574 /-1$ transcriptional regulatory region of JAG1 containing the $-145 /-140$ RUNX3 binding site was responsible for the RUNX3-induced repression of JAG1. Similar results were obtained in Huh7 cells (data not shown).

RUNX3 bound to the transcriptional regulatory region of $J A G 1$. To determine whether RUNX3 binds to the binding site in the JAG1 transcriptional regulatory region, EMSA was performed. Nuclear extracts from Hep3B-CAT and Hep3BRUNX3 cells were analyzed using the RUNX3 binding site as the probe (Fig. 3B). Nuclear extract from RUNX3-expressing Hep3B cells induced the formation of a specific band shift, while the nuclear extract from control CAT-transfected Hep3B 


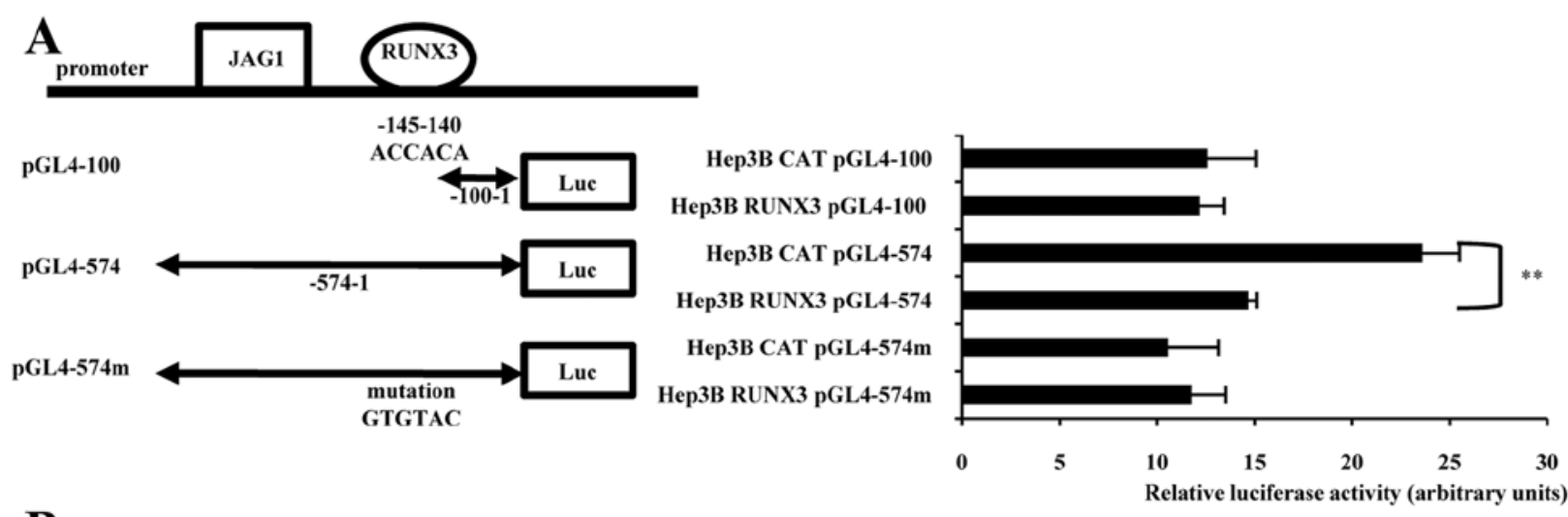

B

\begin{tabular}{|l|c|c|c|c|c|c|c|}
\hline & Lane 1 & Lane 2 & Lane 3 & Lane 4 & Lane 5 & Lane6 & Lane7 \\
\hline wild oligo & + & + & + & + & + & - & + \\
\hline mutant oligo & - & - & - & - & - & + & - \\
\hline competitor wild oligo & - & - & + & - & - & - & - \\
\hline competitor mutant olig0 & - & - & - & - & + & - & - \\
\hline Hep3B-RUNX3 nuclear extracts & - & + & + & + & + & + & - \\
\hline Hep3B-CAT nuclear extracts & - & - & - & - & - & - & + \\
\hline RUNX3 antibody & - & - & - & + & - & - & - \\
\hline
\end{tabular}

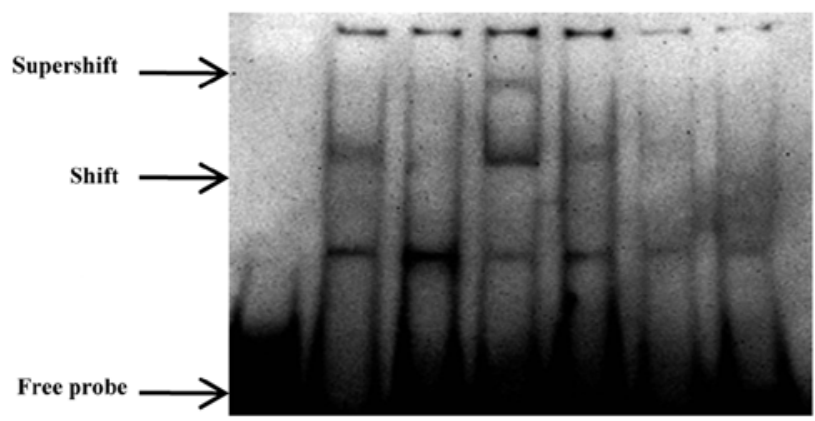

C

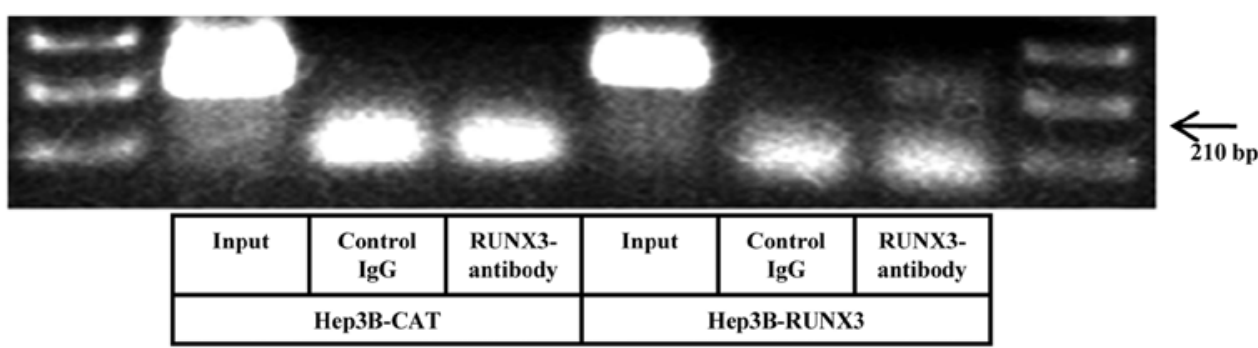

Figure 3. The effect of RUNX3 expression on the JAG1 transcriptional regulatory region. (A) JAG1 transcriptional reporter constructs were transfected into Hep3B cells with RUNX3 or control CAT constructs by using FuGENE6 transfection reagent. Cells were incubated for $48 \mathrm{~h}$, and the cellular extract was then collected. JAG1 transcriptional activity was determined using a luciferase reporter assay. JAG1 transcriptional activity was normalized to the constitutively active Renilla luciferase activity. Data represent the mean $\pm \mathrm{SE}$ of 3 or more independent experiments, each with duplicates. ${ }^{* *} \mathrm{p}<0.01$, statistical significance. (B) Nuclear extracts from Hep3B-CAT and Hep3B-RUNX3 cells were analyzed by EMSA by using oligonucleotides containing wild-type (lanes 1-5 and 7) or mutant (lane 6) RUNX3 binding sites as probes. The identity of the supershift band was examined by incubation with anti-RUNX3 antibody (lane 4). For the inhibition assay, nuclear extract was incubated with an excess of unlabeled wild-type oligonucleotide (lane 3) or mutant oligonucleotide (lane 5). Shown here is a representative blot from 3 independent experiments. (C) Chromatin fragments from Hep3B-CAT or Hep3B-RUNX3 cells were immunoprecipitated overnight with or without specific anti-RUNX3 antibody. PCR was performed to detect a putative RUNX3-binding site in the transcriptional regulatory region of JAG1. The amplified fragment was $210 \mathrm{bp}$. Shown here is a representative blot from 3 independent experiments.

cells did not induce a band shift. Anti-RUNX3 antibody treatment induced a supershift in the DNA-binding complex. These results strongly suggested that RUNX3 bound to the putative RUNX3-binding site of the transcriptional regulatory region of JAG1. Moreover, a ChIP assay was performed to confirm that RUNX3 binds to the putative RUNX3-binding site in the transcriptional regulatory region of JAG1. Chromatin fragments prepared from Hep3B-CAT and Hep3B-RUNX3 cells were immunoprecipitated with or without a specific antiRUNX3 antibody. RUNX3 was found to bind to the putative RUNX3-binding site in the transcriptional regulatory region of JAG1 (Fig. 3C). Therefore, RUNX3 directly regulates JAG1 transcription in a negative manner, suggesting that the loss of RUNX3 expression leads to JAG1 expression. 
A

\begin{tabular}{l|l} 
Hep3B & $\begin{array}{l}\text { Hep3B- } \\
\text { CAT }\end{array}$ \\
\hline $\begin{array}{l}\text { Hep3B- } \\
\text { DAPT }\end{array}$ & $\begin{array}{l}\text { Hep3B- } \\
\text { RUNX3 }\end{array}$
\end{tabular}

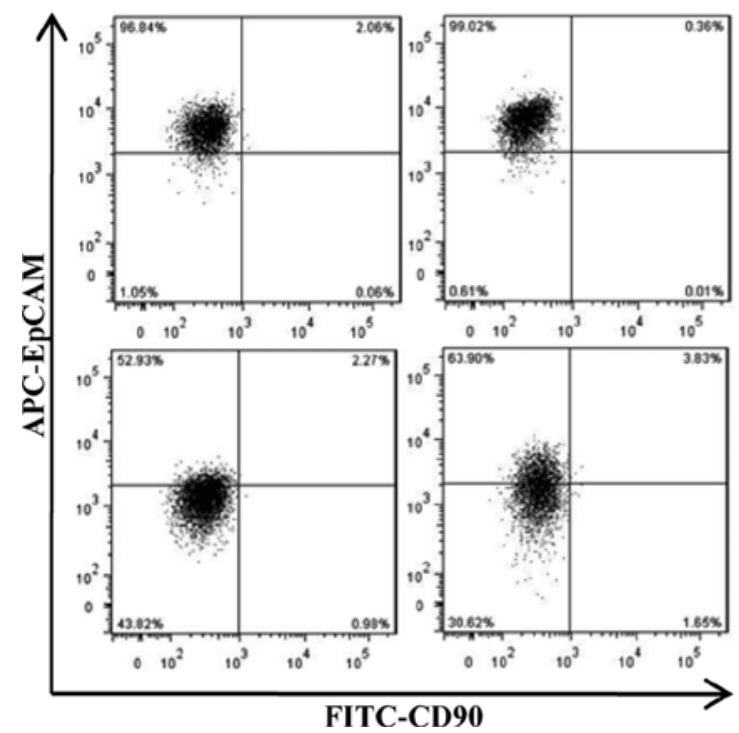

B

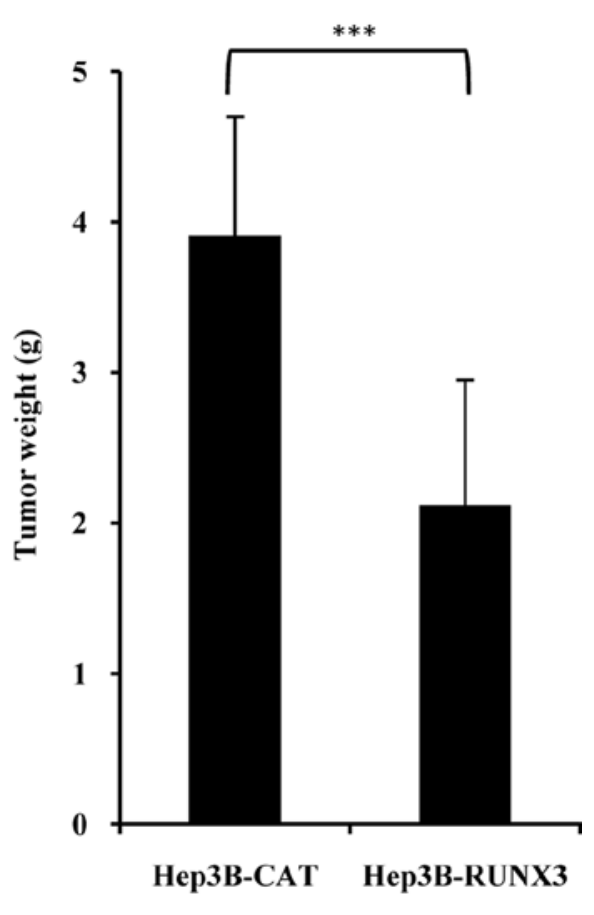

Figure 4. RUNX3 expression reduces the number of CSCs in HCC. (A) Hep3B cells treated with or without DAPT for 2 weeks and Hep3B-CAT and Hep3B-RUNX3 cells were used. Cells were harvested and washed in PBS without $\mathrm{Ca}^{2+} / \mathrm{Mg}^{2+}$ and incubated with APC-labeled anti-EpCAM antibody and FITC-labeled anti-CD90 antibody. The cells were analyzed with a Becton-Dickinson FACS Aria flow cytometer. Shown here are representative plots from 4 or more independent experiments. (B) Hep3B-CAT and Hep3B-RUNX3 cells $\left(2 \times 10^{6}\right)$ were injected into the livers of SCID mice. Tumors were removed 8 weeks after injection, and their gross weights were recorded. Data represent the mean \pm SE of 6 or more independent experiments, each with duplicates. **** $\mathrm{p}<0.001$, statistical significance.

Restoration of RUNX3 reduced EpCAM-positive HCC cells through Notch signaling. A previous study indicated that Notch signaling in HCC may contribute to CSC maintenance (30). We hypothesized that RUNX3 expression reduced the CSC population. To elucidate the change of CSCs in HCC, we examined Hep3B cells incubated with or without DAPT and Hep3B-CAT and Hep3B-RUNX3 cells, which were stained with fluorescence-conjugated primary antibodies against surface markers for putative CSCs (CD44, CD90, CD133 and EpCAM), by flow cytometry. The number of EpCAM-positive cells was reduced in Hep3B cells treated with a $\gamma$-secretase inhibitor and in Hep3B-RUNX3 cells (Fig. 4A). However, there was no change in CSC-like cells stained for CD44, CD90 and CD133 (data not shown). These results indicated that repression of Notch signaling reduced CSC-like cells, which were EpCAM-positive, and RUNX3 reduced CSC-like cells in HCC through Notch signaling repression. Previous studies revealed that HCC that included CSCs had greater tumorigenic ability than HCC that did not include CSCs (31-33). To determine whether restoration of RUNX3 affects tumorigenicity, we constructed mouse xenograft models with Hep3B-CAT and Hep3B-RUNX3. Hep3B-CAT cells initiated tumors in 10 of 11 injected mice, whereas Hep3B-RUNX cells produced tumors in 6 of 11 injected mice 8 weeks after injection. The tumorigenic capacity of Hep3B-CAT cells is higher than that of Hep3B-RUNX3 cells. Moreover, Hep3B-CAT cells produced large hypervascular tumors $(3.94 \pm 0.78 \mathrm{~g})$, whereas Hep3B-RUNX3 cells produced only small hypovascular tumors $(1.88 \pm 0.80 \mathrm{~g})$ (Fig. 4B). Hep3B-CAT cells demonstrated greater tumor-formation ability than Hep3BRUNX3 cells.

\section{Discussion}

In the present study, we demonstrated that the loss of RUNX3 induced cancer stemness through JAG1 activation. RUNX3 is well known as a tumor suppressor in gastric cancer (9). The expression of RUNX3 is decreased in other cancers such as those of the esophagus, colon, lung, pancreas, bile duct and liver $(14,16,17,34,35)$. These results suggest that RUNX3 functions as a tumor suppressor, whereas the precise function of RUNX3 in HCC remains to be elucidated. Microarray analysis of ectopic RUNX3-expressing cells revealed that JAG1 expression was markedly downregulated. JAG-Notch signaling is a candidate signaling target of RUNX3. In the HCC cell lines examined in the present study, JAG1 expression was inversely associated with RUNX3 expression (Fig. 1A). Both RUNX3 and JAG1 were expressed in PLC cells, perhaps because of another regulatory mechanism for JAG1 expression. We assessed JAG1 expression in $\mathrm{HCC}$ tissues and the corresponding tumor-free sections and found that JAG1 was expressed in many HCC tissues $(83.3 \%)$, but not in the corresponding tumor-free sections (43.3\%) (Fig. 1B). The correlation between JAG1 expression and RUNX3 expression was also analyzed in HCC 
tissues by immunohistochemistry. In accordance with the analysis of HCC cell lines, JAG1 expression was inversely associated with RUNX3 expression in HCC tissues (Fig. 1C). RUNX3 was expressed in most of the corresponding tumorfree sections $(86.7 \%)$.

JAG1 is known as a Notch ligand and Notch signaling activator (36). It was reported that Notch signaling dysfunction is related to T-cell leukemia, melanoma, breast cancer and colon cancer $(23,25,37,38)$. However, previous studies reported that Notch in HCC is either an oncogene or a tumor suppressor $(39,40)$. Thus, the role of Notch signaling in HCC is still controversial. JAG1-dependent Notch signaling is dispensable for hematopoietic stem cell self-renewal and differentiation (22). Notch signaling is activated during liver regeneration and potentially contributes to signals affecting cell growth and cell differentiation (41). Therefore, we assessed the effect of ectopic RUNX3 expression on Notch signaling. The restoration of RUNX3 expression suppressed not only JAG1 expression but also Notch signaling (Fig. 2A). The suppression of JAG1 expression contributed to the downregulation of Notch signaling. Notch1 intracellular domain (Notch1IC) and Hes1 expression were suppressed in RUNX3-expressing Hep3B and Huh7 cells (Fig. 2A). These findings indicate that RUNX3 expression regulates JAG1-Notch signaling.

We hypothesized that RUNX3 directly regulates JAG1 expression. To determine whether RUNX3 regulates the expression of JAG1 by directly binding to the transcriptional regulatory region of JAG1, we conducted a JAG1 transcriptional reporter assay, EMSA and ChIP assay. The findings from these assays revealed that RUNX3 directly regulated JAG1 expression by binding to the JAG1 transcriptional regulatory region (Fig. 3A-C). To our knowledge, this is the first report to demonstrate that RUNX3 induces JAG1 suppression through the direct binding of RUNX3 to the transcriptional regulatory region of JAG1.

We next focused on the role of Notch signaling activation in HCC development. Notch signaling is known as one of the major signals required for cancer stemness (30). We hypothesized that the restoration of RUNX3 expression reduced the number of CSCs via the downregulation of Notch signaling. It has been demonstrated that CSCs in HCC can be identified by several cell surface antigens including CD133, CD90, CD44 and EpCAM or by selecting for the side population cells in Hoechst dye-staining $(32,33,42-44)$. The population of EpCAM-positive Hep3B cells was reduced by restoring RUNX3 expression (Fig. 4A), while no significant difference was found in the populations of CD44-positive, CD90-positive, or CD133-positive cells (data not shown). The subset of EpCAM-positive HCC cells was also decreased by inhibiting Notch signaling with a $\gamma$-secretase inhibitor (Fig. 4A). These results suggested that RUNX3 expression reduced the subset of CSCs in HCC via JAG1-Notch signaling suppression. RUNX3 restoration regulated only EpCAM expression and did not have any significant effect on other stem cell markers such as CD44, CD90 and CD133. Further study is necessary to elucidate this.

\section{Acknowledgements}

We thank Tatsuya Fujikawa and Naoki Ueda for their valuable suggestions and Noriaki Tanaka for providing the HCC tissues.

\section{References}

1. El-Serag HB and Rudolph KL: Hepatocellular carcinoma: epidemiology and molecular carcinogenesis. Gastroenterology 132: 2557-2576, 2007.

2. Garcia M, Jernal A, Ward EM, et al: Global Cancer Facts \& Figures 2007.

3. Parkin DM, Pisani P and Ferlay J: Global cancer statistics. CA Cancer J Clin 49: 33-64, 1999.

4. El-Serag HB and Mason AC: Rising incidence of hepatocellular carcinoma in the United States. N Engl J Med 340: 745-750, 1999.

5. Aravalli RN, Steer CJ and Cressman EN: Molecular mechanisms of hepatocellular carcinoma. Hepatology 48: 2047-2063, 2008.

6. Nishida N, Nagasaka T, Nishimura T, Ikai I, Boland CR and Goel A: Aberrant methylation of multiple tumor suppressor genes in aging liver, chronic hepatitis, and hepatocellular carcinoma. Hepatology 47: 908-918, 2008.

7. Yeh SH, Chen PJ, Chen HL, Lai MY, Wang CC and Chen DS: Frequent genetic alterations at the distal region of chromosome 1p in human hepatocellular carcinomas. Cancer Res 54: 4188-4192, 1994.

8. Piao Z, Park C, Park JH and Kim H: Allelotype analysis of hepatocellular carcinoma. Int J Cancer 75: 29-33, 1998.

9. Li QL, Ito K, Sakakura C, et al: Causal relationship between the loss of RUNX3 expression and gastric cancer. Cell 109: 113-124, 2002.

10. Fukamachi H, Ito $\mathrm{K}$ and Ito $\mathrm{Y}$ : Run $\times 3^{-/}$gastric epithelial cells differentiate into intestinal type cells. Biochem Biophys Res Commun 321: 58-64, 2004

11. Yamamura Y, Lee WL, Inoue K, Ida H and Ito Y: RUNX3 cooperates with FoxO3a to induce apoptosis in gastric cancer cells. J Biol Chem 281: 5267-5276, 2006.

12. Guo WH, Weng LQ, Ito K, et al: Inhibition of growth of mouse gastric cancer cells by Runx3, a novel tumor suppressor. Oncogene 21: 8351-8355, 2002.

13. Kim TY, Lee HJ, Hwang KS, et al: Methylation of RUNX3 in various types of human cancers and premalignant stages of gastric carcinoma. Lab Invest 84: 479-484, 2004.

14. Ku JL, Kang SB, Shin YK, et al: Promoter hypermethylation downregulates RUNX3 gene expression in colorectal cancer cell lines. Oncogene 23: 6736-6742, 2004.

15. Yanada M, Yaoi T, Shimada J, et al: Frequent hemizygous deletion at 1 p36 and hypermethylation downregulate RUNX3 expression in human lung cancer cell lines. Oncol Rep 14: $817-822,2005$.

16. Wada M, Yazumi S, Takaishi S, et al: Frequent loss of RUNX3 gene expression in human bile duct and pancreatic cancer cell lines. Oncogene 23: 2401-2407, 2004.

17. Mori T, Nomoto S, Koshikawa K, et al: Decreased expression and frequent allelic inactivation of the RUNX3 gene at $1 \mathrm{p} 36$ in human hepatocellular carcinoma. Liver Int 25: 380-388, 2005.

18. Artavanis-Tsakonas S, Rand MD and Lake RJ: Notch signaling: cell fate control and signal integration in development. Science 284: 770-776, 1999.

19. Fiuza UM and Arias AM: Cell and molecular biology of Notch. J Endocrinol 194: 459-474, 2007.

20. Roy M, Pear WS and Aster JC: The multifaceted role of Notch in cancer. Curr Opin Genet Dev 17: 52-59, 2007.

21. Radtke F and Raj K: The role of Notch in tumorigenesis: oncogene or tumour suppressor? Nat Rev Cancer 3: 756-767, 2003.

22. Mancini SJ, Mantei N, Dumortier A, Suter U, MacDonald HR and Radtke F: Jagged1-dependent Notch signaling is dispensable for hematopoietic stem cell self-renewal and differentiation. Blood 105: 2340-2342, 2005.

23. Ayyanan A, Civenni G, Ciarloni L, et al: Increased Wnt signaling triggers oncogenic conversion of human breast epithelial cells by a Notch-dependent mechanism. Proc Natl Acad Sci USA 103: 3799-3804, 2006.

24. van Es JH, van Gijn ME, Riccio O, et al: Notch/gammasecretase inhibition turns proliferative cells in intestinal crypts and adenomas into goblet cells. Nature 435: 959-963, 2005.

25. Balint K, Xiao M, Pinnix CC, et al: Activation of Notch1 signaling is required for beta-catenin-mediated human primary melanoma progression. J Clin Invest 115: 3166-3176, 2005.

26. Lapidot T, Sirard C, Vormoor J, et al: A cell initiating human acute myeloid leukaemia after transplantation into SCID mice. Nature 367: 645-648, 1994.

27. Wicha MS, Liu S and Dontu G: Cancer stem cells: an old idea-a paradigm shift. Cancer Res 66: 1883-1896, 2006. 
28. Zeng Q, Li S, Chepeha DB, et al: Crosstalk between tumor and endothelial cells promotes tumor angiogenesis by MAPK activation of Notch signaling. Cancer Cell 8: 13-23, 2005.

29. Zhao F, Xuan Z, Liu L and Zhang MQ: TRED: a transcriptional regulatory element database and a platform for in silico gene regulation studies. Nucleic Acids Res 33: D103-D107, 2005.

30. Mishra L, Banker T, Murray J, et al: Liver stem cells and hepatocellular carcinoma. Hepatology 49: 318-329, 2009.

31. Suetsugu A, Nagaki M, Aoki H, Motohashi T, Kunisada T and Moriwaki H: Characterization of $\mathrm{CD} 133^{+}$hepatocellular carcinoma cells as cancer stem/progenitor cells. Biochem Biophys Res Commun 351: 820-824, 2006.

32. Yamashita T, Ji J, Budhu A, et al: EpCAM-positive hepatocellular carcinoma cells are tumor-initiating cells with stem/ progenitor cell features. Gastroenterology 136: 1012-1024, 2009.

33. Yang ZF, Ho DW, Ng MN, et al: Significance of CD90 $0^{+}$cancer stem cells in human liver cancer. Cancer Cell 13: 153-166, 2008.

34. Sakakura C, Miyagawa K, Fukuda KI, et al: Frequent silencing of RUNX3 in esophageal squamous cell carcinomas is associated with radioresistance and poor prognosis. Oncogene 26 5927-5938, 2007.

35. Li QL, Kim HR, Kim WJ, et al: Transcriptional silencing of the RUNX3 gene by CpG hypermethylation is associated with lung cancer. Biochem Biophys Res Commun 314: 223-228, 2004.

36. Lindsell CE, Shawber CJ, Boulter J and Weinmaster G: Jagged: a mammalian ligand that activates Notch1. Cell 80: 909-917, 1995.
37. Fre S, Huyghe M, Mourikis P, Robine S, Louvard D and Artavanis-Tsakonas S: Notch signals control the fate of immature progenitor cells in the intestine. Nature 435: 964-968, 2005.

38. Weng AP, Ferrando AA, Lee W, et al: Activating mutations of NOTCH1 in human T cell acute lymphoblastic leukemia. Science 306: 269-271, 2004.

39. Cantarini MC, de la Monte SM, Pang M, et al: Aspartylasparagyl beta hydroxylase over-expression in human hepatoma is linked to activation of insulin-like growth factor and notch signaling mechanisms. Hepatology 44: 446-457, 2006.

40. Qi R, An H, Yu Y, et al: Notch1 signaling inhibits growth of human hepatocellular carcinoma through induction of cell cycle arrest and apoptosis. Cancer Res 63: 8323-8329, 2003.

41. Kohler C, Bell AW, Bowen WC, Monga SP, Fleig W and Michalopoulos GK: Expression of Notch-1 and its ligand Jagged-1 in rat liver during liver regeneration. Hepatology 39: 1056-1065, 2004

42. Ma S, Chan KW, Hu L, et al: Identification and characterization of tumorigenic liver cancer stem/progenitor cells. Gastroenterology 132: 2542-2556, 2007.

43. Yang ZF, Ngai P, Ho DW, et al: Identification of local and circulating cancer stem cells in human liver cancer. Hepatology 47: 919-928, 2008

44. Chiba T, Kita K, Zheng YW, et al: Side population purified from hepatocellular carcinoma cells harbors cancer stem cell-like properties. Hepatology 44: 240-251, 2006. 